\title{
Modelagem numérica da transferência de calor do processo de lingotamento contínuo de tarugo oco de cobre em uma planta industrial
}

\author{
Edson Fouad Beck Filho \\ Paulo Jesus Aniceto ${ }^{1}$ \\ Noé Cheung ${ }^{2}$ \\ Carlos Henrique da Silva Santos \\ Felipe Bertelli ${ }^{1,4 *}$ (1)
}

\section{Resumo}

O objetivo do trabalho é apresentar uma metodologia numérica envolvendo o modelamento matemático da transferência de calor radial, baseado no Método das Diferenças Finitas, para simular a solidificação de tarugos cilíndricos ocos de cobre puro durante a passagem pelo molde primário de refrigeração. Foi utilizado o método do problema inverso em condução de calor aplicado à solidificação para determinação do coeficiente global de transferência de calor na interface metal/molde $\left(\mathrm{h}_{\mathrm{g}}\right)$ considerando cinco temperaturas experimentais em posições distintas da superfície do lingote, para uma corrida com velocidade de $180 \mathrm{~mm} / \mathrm{min}$. Posteriormente, foram estimadas, via simulação numérica, as distribuições internas de temperatura e gerados os perfis térmicos bidimensionais no sentido longitudinal de lingotamento, bem como da secção transversal ao sentido de lingotamento. Os resultados evidenciaram uma heterogeneidade na troca térmica entre o lingote e o molde de refrigeração, considerando diferentes posições angulares em relação a secção transversal de lingotamento, indicando a maior eficiência na transferência de calor na região inferior do molde e o oposto na região superior.

Palavras-chave: Lingotamento contínuo; Modelagem numérica; Método das diferenças finitas.

\section{Numerical modeling of the continuous casting hollow billets heat transfer in industrial plant}

\begin{abstract}
s
The objective of the work is to present a numerical methodology involving the mathematical modeling of the radial heat transfer, based on the Finite Differences Method, to simulate the solidification of pure copper hollow billets during their passage through the primary cooling region at the mold of a continuous casting equipment. The inverse problem method in heat conduction applied to solidification was used to determine the global heat transfer coefficient at the metal $/ \mathrm{mold}$ interface $\left(\mathrm{h}_{\mathrm{g}}\right)$ basing on five experimental surface temperatures at different positions, considering a casting speed of 180 $\mathrm{mm} / \mathrm{min}$. Subsequently, inner temperature distributions were estimated using numerical simulation and the two-dimensional thermal profiles were generated for the longitudinal casting direction, as well as for the cross section to casting direction. The results showed a heterogeneity in the thermal exchange between the ingot and the cooling mold, considering different angular positions in relation to the cross section of the casting, indicating greater efficiency in the lower region of the mold and the opposite side at the upper region.
\end{abstract}

Keywords: Continuous casting; Numerical modeling; Finite difference method.

${ }^{1}$ Universidade Santa Cecilia - UNISANTA, Santos, SP, Brasil.

${ }^{2}$ Universidade Estadual de Campinas - UNICAMP, Campinas, SP, Brasil.

${ }^{3}$ Instituto Federal de Educação, Ciência e Tecnologia de São Paulo-IFSP, Itapetininga, SP, Brasil.

${ }^{4}$ Universidade Federal de São Paulo - UNIFESP, Santos, SP, Brasil.

*Autor correspondente: bertelli@unisanta.br; bertelli@unifesp.br

2176-1523 C 2021. Beck Filho et al. Publicado pela ABM. Este é um artigo publicado em acesso aberto (Open Access) sob a licença Creative Commons Attribution, que permite uso, distribuição e reprodução em qualquer meio, sem restrições desde que o trabalho original seja corretamente citado. 


\section{Introdução}

A aplicação da modelagem matemática na análise da transferência de calor durante a solidificação de metais, especificamente em processos de lingotamento contínuo, é uma importante ferramenta para auxiliar o setor industrial no controle dos parâmetros operacionais de equipamentos vinculados ao resfriamento, que influenciam na qualidade final de seu produto e nas relações custo-benefício de fabricação [1-3]. É possível prever as propriedades mecânicas de fundidos conhecendo-se os parâmetros térmicos envolvidos na solidificação, os quais definem o arranjo micro e macroestrutural resultantes da transformação líquido/sólido [4].

Em pesquisas sobre a produção de tarugos de cobre proveniente do processo de lingotamento contínuo, a literatura apresenta estudos numéricos evidenciando os efeitos dos parâmetros térmicos de solidificação sobre o crescimento de grãos, indicando a indução magnética no molde como principal abordagem para o refino de grãos [5-7]. Porém, estes estudos se baseiam em modelos tridimensionais e sofisticados equacionamentos do ponto de vista fluidodinâmico, os quais normalmente demandam alto custo computacional de armazenamento e processamento de dados devido ao número de equações que devem ser resolvidas em cada nó da malha.

Há também autores que caracterizam o comportamento do fluxo de calor na interface metal $/$ molde nos processos de solidificação como sendo o maior fator de relevância para o controle do tamanho de grão e de suas propriedades mecânicas $[8,9]$.

Em ambos os casos, do ponto de vista da segurança operacional do processo de lingotamento contínuo, a falta de controle do avanço da interface líquido/sólida pode gerar vazamentos de metal fundido e interromper o processo, causando paradas inesperadas e custos adicionais.

Neste contexto, este trabalho tem por objetivo modelar numericamente a transferência de calor durante o processo de solidificação de cobre puro durante a passagem pelo molde primário de refrigeração de um equipamento industrial de lingotamento contínuo horizontal de tarugos cilíndricos ocos. A equação geral da condução de calor e o balanço energético são utilizados para simular a transferência de calor de forma unidirecional ao longo do raio do lingote, aplicando-se o Método de Diferenças Finitas, a fim de determinar o mapeamento térmico no interior do tarugo durante a transformação de fase líquido/sólida. Inicialmente é simulado o coeficiente de transferência de calor na interface metal/molde $\left(\mathrm{h}_{\mathrm{g}}\right)$, em 5 posições da superfície do lingote, pelo Método do Problema Inverso de Condução de Calor.

Por fim, as temperaturas simuladas para as 5 posições são interpoladas linearmente dentro de cada setor limitado por duas dessas posições adjacentes, a fim de se obter a distribuição de temperatura ao longo de toda a área da secção transversal do lingote. A avaliação do avanço da interface líquido/sólido é realizada através do mapeamento térmico bidimensional longitudinal, pela composição destas várias secções transversais ao longo do tempo.

\section{Materiais e métodos}

\subsection{Procedimento experimental}

O procedimento experimental para a obtenção dos dados térmicos de uma corrida de lingotamento contínuo horizontal de cobre fosforado (Liga comercial UNS C12200, com percentual de fósforo menor que $0,02 \%$ em peso) foi realizado em uma planta industrial localizada no $\mathrm{ABC}$ Paulista. O procedimento experimental do registro foto térmico na saída do molde de refrigeração, utilizando uma câmera termográfica da marca Fluke Ti32, está esquematizado na Figura 1.

A partir da imagem termográfica da superfície do tarugo, foram identificadas 05 posições distribuídas $45^{\circ}$ umas das outras, desde o topo até a base da secção transversal. $\mathrm{O}$ equipamento de lingotamento horizontal produz um tarugo oco cilíndrico, com um raio externo de $0,0508 \mathrm{~m}$ e um furo

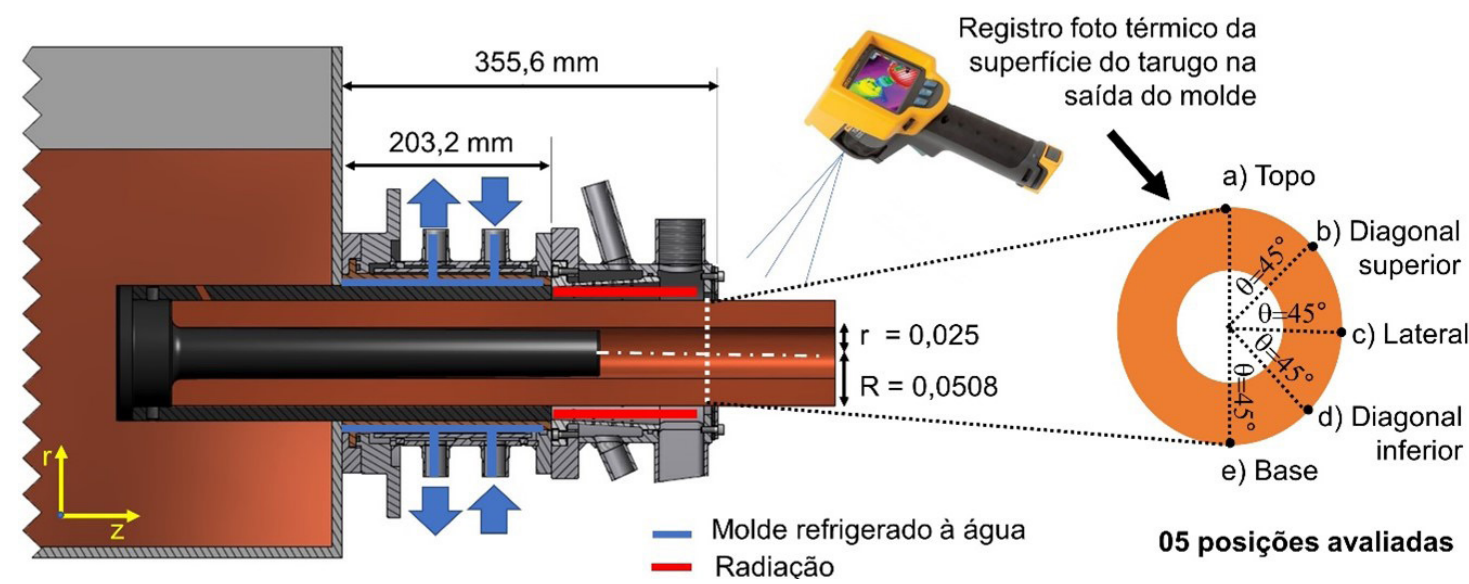

Figura 1. Regiões de resfriamento dentro do molde e registro termográfico na saída do molde, com identificação das 05 posições avaliadas na simulação numérica. 
central com raio interno de $0,025 \mathrm{~m}$. Durante a produção do lingote, a extração de calor ocorre por um molde de grafite, que é refrigerado à água nos primeiros 203,2 mm.

Na sequência, o tarugo passa por uma região de radiação em que a troca térmica é ineficiente, uma vez que o ar (aquecido rapidamente com a passagem do fundido) ao redor da superfície está enclausurado naquela região, conforme indicado pela linha vermelha da Figura 1. Em condições operacionais, esta região possui um preenchimento de água para continuidade do resfriamento. Entretanto, para evitar distorções no registro térmico logo à frente na saída do molde, para este estudo a água foi desligada nesta segunda região e esperou-se ao menos 10 segundos para obtenção das imagens termográficas. Assim, nota-se que o fluxo térmico ocorre radialmente para o molde refrigerado, enquanto ao centro há um molde de grafite não refrigerado, que serve de guia para a conformação do furo deste tarugo oco.

\subsection{Modelagem numérica em diferenças finitas para solidificação radial de tarugo cilíndrico oco}

A determinação da variação de temperatura em um meio discreto, em função do tempo e da posição, pode ser modelada a partir da equação geral da condução de calor [10] que, em um sistema de coordenadas cilíndricas com fluxo de calor radial, sem trocas térmicas no sentido longitudinal (eixo z) ou angular $(\phi)$, pode ser representado pela Equação 1, sendo $k$ a condutividade térmica $(\mathrm{W} / \mathrm{m} . \mathrm{K})$, c o calor específico (J/kg.K), $\rho$ a massa específica $\left(\mathrm{kg} / \mathrm{m}^{3}\right), \mathrm{T}$ a temperatura (K), t o tempo (s) e r o raio (m). Aplicando-se a Equação 1 em Diferenças Finitas:

$$
\frac{1}{r} \cdot \frac{\partial}{\partial r} \cdot\left(k \cdot r \cdot \frac{\partial T}{\partial r}\right)=\rho \cdot c \cdot \frac{\partial T}{\partial t}
$$

e isolando-se o termo $\mathrm{T}_{\mathrm{i}}^{\mathrm{n}+1}$ obtém-se a Equação 2 que define a temperatura no instante de tempo atual $(n+1)$, para

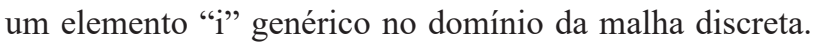
$\mathrm{O}$ índice (n) refere-se ao tempo anterior e $\Delta \mathrm{r}$ é a malha espacial.

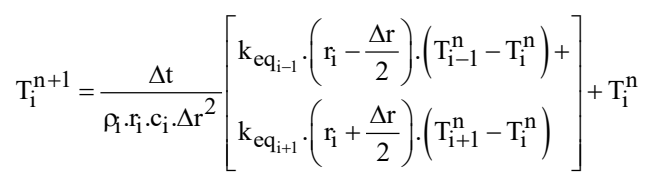

Foi considerada uma condutividade térmica equivalente $\left(\mathrm{k}_{\mathrm{eq}} \mathrm{it+}\right)$ entre dois elementos adjacentes, sendo dada pela Equação 3:

$$
\mathrm{k}_{\mathrm{eq}}=\frac{2 \cdot \mathrm{k}_{\mathrm{i}+1} \cdot \mathrm{k}_{\mathrm{i}}}{\mathrm{k}_{\mathrm{i}+1}+\mathrm{k}_{\mathrm{i}}}
$$

A variação espacial da malha em Diferenças Finitas se inicia no molde interno de grafite central (usado para conformação do furo no interior) e vai até a superfície exterior do tarugo de cobre, onde ocorre a troca térmica com o molde externo, de acordo com a Figura 2. Nesse caso, tem-se: o

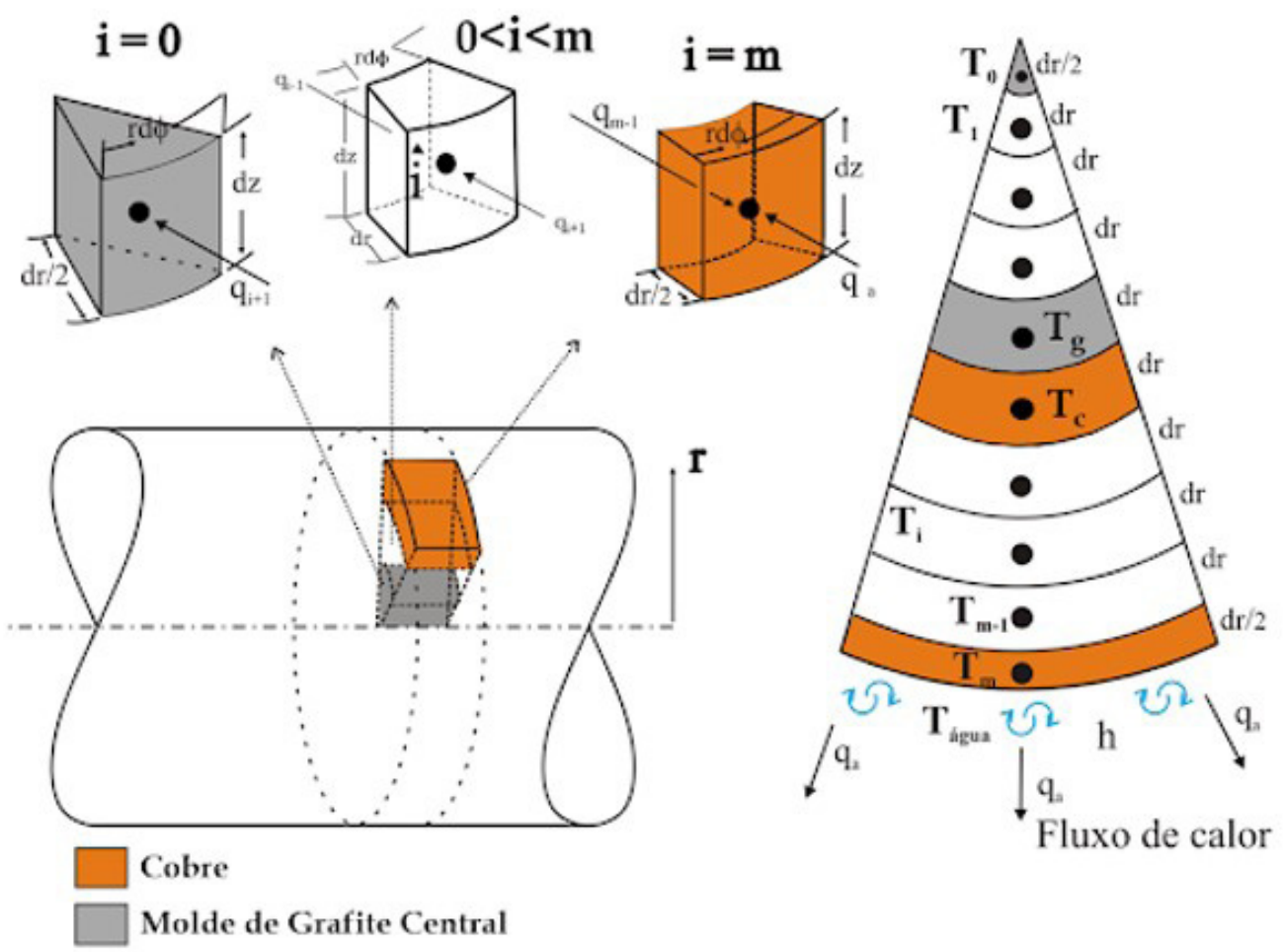

Figura 2. Balanço térmico nos elementos de fronteira $\mathrm{i}=0$ e $\mathrm{i}=\mathrm{m}$, e elemento genérico $0<\mathrm{i}<\mathrm{m}$. 
elemento de malha $\mathrm{i}=0$ como sendo o primeiro elemento do molde central de grafite; $\mathrm{i}=\mathrm{g}$ o último elemento do molde central; $\mathrm{i}=\mathrm{c}$ o primeiro elemento de cobre que faz contato com o molde de grafite central e $\mathrm{i}=\mathrm{m}$ representando a condição de fronteira na interface metal/molde de refrigeração. A Equação 2 é aplicada a todos os elementos de malha compreendidos entre $\mathrm{i}=0$ e $\mathrm{i}=\mathrm{m}$, ou seja, $0<\mathrm{i}<\mathrm{m}$.

Já para a região de interface metal/molde refrigerado $(\mathrm{i}=\mathrm{m})$, a equação em diferenças finitas pode ser obtida aplicando-se o balanço térmico no elemento, conforme a Equação 4 tendo $h_{g}$ como sendo o coeficiente global de transferência de calor na interface metal $/$ molde $\left(\mathrm{W} \cdot \mathrm{m}^{-2} \mathrm{~K}^{-1}\right)$, o qual leva em conta a resistência térmica da água, do molde e do gap de ar devido à contração da solidificação:

$$
h_{g} \cdot A_{m} \cdot\left(T_{a}-T_{m}\right)+\left.k_{e q_{m-1}} \cdot A_{m-1} \cdot \frac{\partial T}{\partial r}\right|_{i=m-1}=\left.\rho_{m} \cdot c_{m} \cdot V_{m} \cdot \frac{\partial T}{\partial t}\right|_{i=m}
$$

Com isso, obtém-se a temperatura no instante de tempo posterior a uma variação de tempo $\Delta t$ no elemento de fronteira $\mathrm{i}=\mathrm{m}$, conforme a Equação 5:

$$
T_{m}^{n+1}=\frac{2 . \Delta t}{\rho_{m} \cdot c_{m} \cdot r_{m} \cdot \Delta r} \cdot\left[h_{g} \cdot r_{m} \cdot\left(T_{a}-T_{m}^{n}\right)+k_{e q_{m-1}} \cdot\left(r_{m}-\frac{\Delta r}{2}\right) \cdot\left(T_{m-1}^{n}-T_{m}^{n}\right)\right]+T_{m}^{n}
$$

Por fim, para o primeiro elemento de malha dentro do molde de grafite central (i=0), a Equação 1 apresenta uma inconsistência no termo $1 / \mathrm{r}$ quando $\mathrm{r}$ tende a zero.

Aplicando-se a regra de L'Hôpital e o Método de Diferenças Finitas, este termo passa a ser definido termicamente no Domínio Espacial, chegando-se ao termo isolado $T_{0}^{n+1}$, que representa a temperatura no elemento central no tempo atual, como segue na Equação 6:

$$
T_{0}^{n+1}=\frac{4 \cdot k_{e q} \cdot \Delta t}{\rho_{0} \cdot c_{0} \cdot \Delta r^{2}} \cdot\left(T_{1}^{n}-T_{0}^{n}\right)+T_{0}^{n}
$$

Na Tabela 1 são apresentadas as propriedades termofísicas do cobre puro a ser solidificado e do molde de grafite empregadas na simulação. O material lingotado foi o cobre fosforado, liga comercial UNS C12200, que possui menos de $0,02 \%$ em peso de fósforo, sendo, portanto, muito diluído, permitindo que o modelo de simulação de solidificação de metal puro possa ser aplicado.

O Método de Dusinberre foi utilizado para o controle do avanço da interface líquido/sólido, que considera a relação $\lambda=\mathrm{L} / \mathrm{c}$, que significa uma variação de temperatura equivalente ao calor latente liberado durante a solidificação $[13,14]$.

O cobre líquido dentro dos fornos de alimentação do lingotamento é mantido à $1167^{\circ} \mathrm{C}$, valor este considerado como a temperatura inicial de vazamento na simulação.
As malhas temporal e espacial adotadas em diferenças finitas foram $\Delta \mathrm{t}=0,000025 \mathrm{~s}$ e $\Delta \mathrm{r}=0,0001 \mathrm{~m}$, respectivamente. A velocidade de lingotamento foi de $180 \mathrm{~mm} / \mathrm{min}$.

A determinação do valor de $h_{g}$ foi feita mediante resolução do problema inverso da condução de calor (do inglês Inverse Heat Conduction Problem - IHCP) [15] onde o perfil térmico simulado para um elemento da superfície do cobre $(\mathrm{i}=\mathrm{m})$ inicia-se com a temperatura de vazamento e termina com a temperatura medida por foto termografia imediatamente na saída do molde. Foram escolhidas 5 posições para o cálculo de $\mathrm{h}_{\mathrm{g}}$, uniformemente distribuídas sobre a superfície do molde, localizadas no a) topo, b) diagonal superior a $45^{\circ}$ do topo, c) lateral $\left(90^{\circ}\right.$ a partir do topo), d) diagonal inferior ( $45^{\circ}$ da lateral) e e) base (Figura 1). Além disso, o valor de $h_{g}$ foi determinado em duas etapas distintas: um valor simulado pelo método inverso (IHCP) para a região de contato com o molde refrigerado, durante os primeiros 0,2032 metros de lingotamento (Figura 1), e outro valor fixado em $35 \mathrm{~W} / \mathrm{m}^{2} \mathrm{~K}$ após essa distância até o final do molde. Considerando-se que o fluxo de ar dentro da segunda etapa do molde é restrito (por estar enclausurado e sem movimentos convectivos com o ambiente) e que este se aquece rapidamente pela radiação emitida durante a passagem do lingote por esta região, esse valor adotado refere-se a resfriamentos de superfícies de cobre ao ar sem convecção [16].

Posteriormente, a distribuição de temperatura ao longo do raio do lingote foi determinada e, a fim de se estimar mapeamento térmico em toda área da secção transversal, 50 pontos entre cada uma das 5 posições simuladas foram proporcionalmente distribuídas, segundo uma progressão aritmética de razão $\mathrm{R}=\Delta \mathrm{T} / 50$, onde $\Delta \mathrm{T}$ é a diferença de temperatura entre dois pontos simulados consecutivamente, sendo: entre o topo e diagonal superior, diagonal superior e lado, lado e diagonal inferior e diagonal inferior e base, totalizando 200 elementos de malha em um semicírculo. Finalmente, considerando uma simetria com relação ao diâmetro traçado entre o topo e a base, a secção transversal

\begin{tabular}{|c|c|c|c|}
\hline Propriedades & $\begin{array}{l}\text { Símbolo/ } \\
\text { Unidades }\end{array}$ & Cobre & Grafite \\
\hline \multirow[t]{3}{*}{ Condutividade Térmica } & $\mathrm{k}\left(\mathrm{W} \cdot \mathrm{m}^{-1} \cdot \mathrm{K}^{-1}\right)$ & - & 174,5 \\
\hline & $\mathrm{k}_{\mathrm{S}}$ & 330 & - \\
\hline & $\mathrm{k}_{\mathrm{L}}$ & 166 & - \\
\hline \multirow[t]{3}{*}{ Calor Específico } & $\mathrm{c}\left(\mathrm{J} \cdot \mathrm{kg}^{-1} \cdot \mathrm{K}^{-1}\right)$ & - & 670 \\
\hline & $\mathrm{c}_{\mathrm{s}}$ & 490 & - \\
\hline & $\mathrm{c}_{\mathrm{L}}$ & 494 & - \\
\hline \multirow[t]{2}{*}{ Massa Específica } & $\rho_{\mathrm{S}}\left(\mathrm{kg} \cdot \mathrm{m}^{-3}\right)$ & 8.382 & 1.890 \\
\hline & $\rho_{\mathrm{L}}$ & 7.938 & - \\
\hline Calor Latente Fusão & $\mathrm{L}\left(\mathrm{J} \cdot \mathrm{kg}^{-1}\right)$ & 205.000 & - \\
\hline Temperatura de Fusão & $\mathrm{T}_{\mathrm{f}}(\mathrm{K})$ & 1.356 & - \\
\hline
\end{tabular}
ao sentido de lingotamento completa pode ser representada.

Tabela 1. Propriedades termofísicas do cobre e do grafite $[11,12]$ 


\section{Resultados}

A Figura 3 apresenta as cinco temperaturas experimentais identificadas sobre a superfície do tarugo de cobre logo na saída do molde. A temperatura de $240,70^{\circ} \mathrm{C}$ foi medida no topo, em seguida, $197^{\circ} \mathrm{C}$ na diagonal superior, $167,80^{\circ} \mathrm{C}$ na lateral, $162,30^{\circ} \mathrm{C}$ na diagonal inferior e $161,00^{\circ} \mathrm{C}$ na base. Cada valor foi medido cinco vezes na mesma posição e a indicação refere-se ao valor médio destas medições em cada região. Além disso, o erro de medição pelo equipamento é menor que $2 \%$, conforme indicação do fabricante para a distância e ângulo utilizados nessa medição [17]. Há uma clara tendência de decréscimo de temperatura à medida que são tomadas posições a partir do topo em direção à base.
Considerando-se as temperaturas experimentais da superfície do lingote na saída do molde, foi possível solucionar o problema inverso de condução de calor para determinar $h_{g}$, assim como simular numericamente o perfil de temperatura correspondente a todos os elementos de malha.

$\mathrm{Na}$ Figura 4 observa-se que no instante de tempo $\mathrm{t}=118,50 \mathrm{~s}$, o valor da temperatura simulada coincide com o valor da temperatura experimental, para os seguintes valores de $h_{\mathrm{g}}: 2.767 \mathrm{~W} / \mathrm{m}^{2} \mathrm{~K}$ (topo); $3.107 \mathrm{~W} / \mathrm{m}^{2} \mathrm{~K}$ (diagonal superior); $3.395 \mathrm{~W} / \mathrm{m}^{2} \mathrm{~K}$ (lateral); $3.457 \mathrm{~W} / \mathrm{m}^{2} \mathrm{~K}$ (diagonal inferior) e $3.471,20 \mathrm{~W} / \mathrm{m}^{2} \mathrm{~K}$ (base). No instante de tempo $\mathrm{t}=67,73 \mathrm{~s}$ é possível observar uma inflexão em todas as curvas de resfriamento, indicando que neste ponto o valor de $\mathrm{h}_{\mathrm{g}}$ sofre uma alteração abrupta para $35 \mathrm{~W} / \mathrm{m}^{2} \mathrm{~K}$, na zona
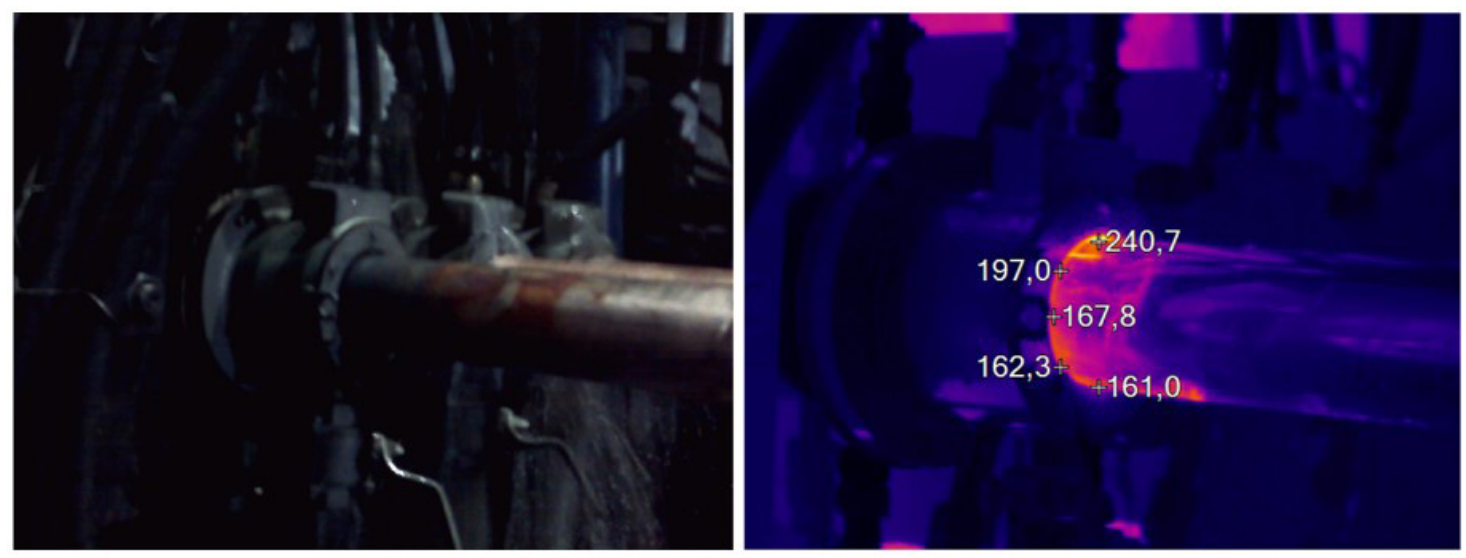

Figura 3. Visão normal e termográfica na saída do molde refrigerado para a velocidade de lingotamento de $180 \mathrm{~mm} / \mathrm{min}$.

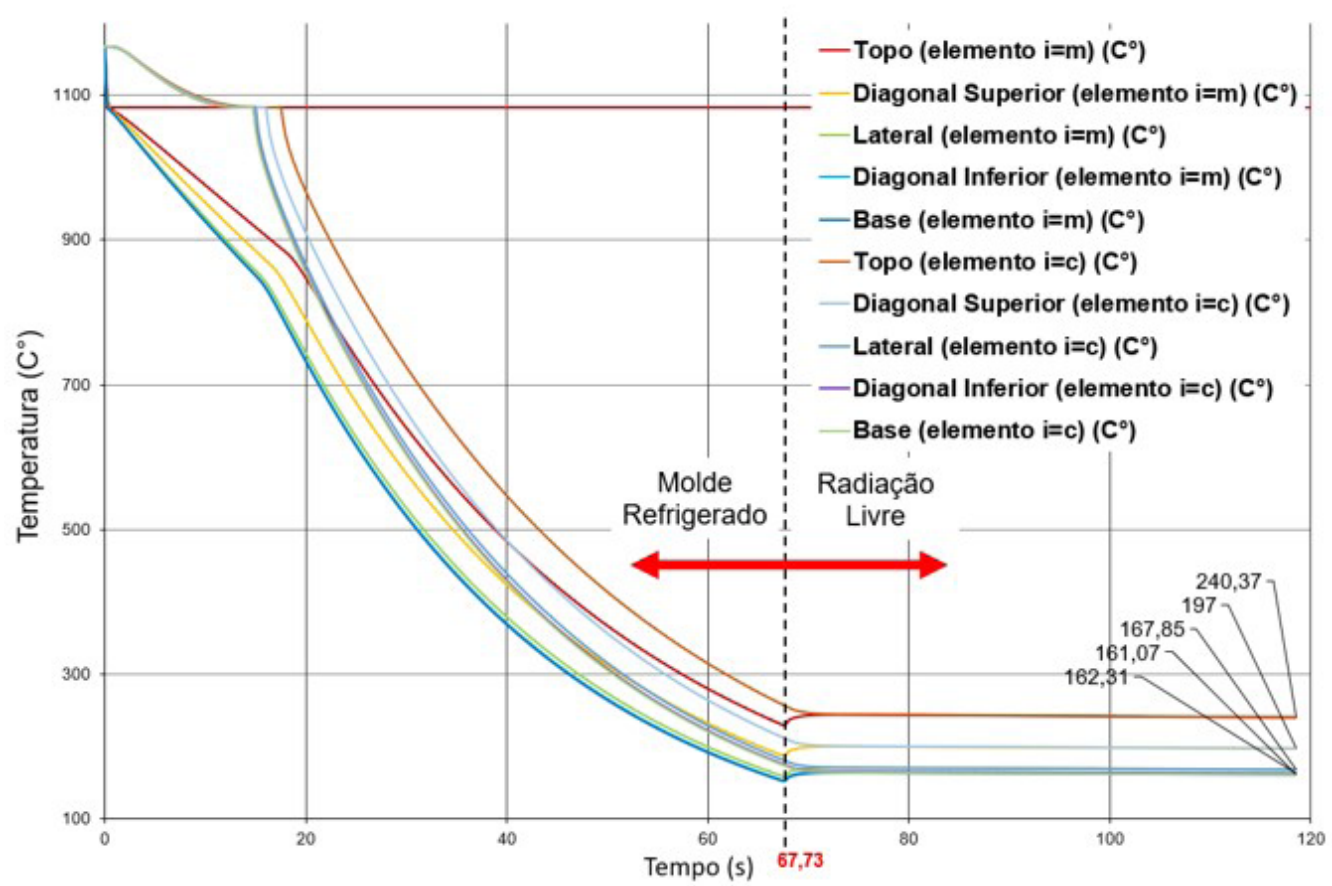

Figura 4. Perfil térmico para a superfície do cobre (elemento $\mathrm{i}=\mathrm{m}$ ) e para o último elemento solidificado $(\mathrm{i}=\mathrm{c})$. 
de radiação. Consequentemente, as temperaturas tendem a permanecer num patamar aproximadamente constante até o final da passagem pelo molde.

A partir da determinação dos valores do coeficiente de transferência de calor na interface metal/molde refrigerado $\left(h_{\mathrm{g}}\right)$, foi possível determinar o comportamento térmico no interior do lingote. Na Figura 5 é possível observar a distribuição de temperatura ao longo da secção longitudinal de lingotamento, em que a linha branca contínua indica a interface líquido/sólido ao longo do tempo.

Quando o tarugo percorre uma distância dentro do molde de 44,25 mm, na direção z de lingotamento, a interface líquido/ sólido toca o molde central no tempo $\mathrm{t}=14,75 \mathrm{~s}$, no ponto da base. Na parte superior ou topo (ponto a), a interface líquido/sólido atinge o molde de grafite central em $\mathrm{z}=52,50 \mathrm{~mm}$, no instante de tempo $t=17,50 \mathrm{~s}$, sendo essa a última camada a solidificar. Nos nas regiões intermediárias entre o topo e a base, para a posição lateral (ponto c) essa interface atinge o molde central em $z=45,00 \mathrm{~mm}$, no tempo $\mathrm{t}=15,00 \mathrm{~s}$. Nas diagonais superior e inferior (pontos b e d), respectivamente, a solidificação completa ocorreu em $\mathrm{z}=48,00 \mathrm{~mm}$, no tempo $\mathrm{t}=16,00 \mathrm{~s}$, e $\mathrm{z}=44,25 \mathrm{~mm}$ no instante de tempo de $14,75 \mathrm{~s}$.
Na Figura 6a, a secção transversal do lingote indica a distribuição das isotermas com os dados interpolados para a posição $\mathrm{z}=44,25 \mathrm{~mm}$, no tempo $\mathrm{t}=14,75 \mathrm{~s}$. Nota-se que apesar da região inferior (ponto e) já estar completamente solidificada, uma parcela na região superior (ponto a) ainda se encontra fundida próximo ao molde central. Somente após $2,75 \mathrm{~s}$ de lingotamento é que a solidificação completa ocorre, de acordo com a Figura $6 b$.

\section{Discussão}

Ao serem tomadas posições a partir do topo do lingote na Figura 4, há um decaimento no valor das temperaturas medidas experimentalmente em direção a base, com uma amplitude total de $79^{\circ} \mathrm{C}$ entre a base e o topo.

Nessas duas regiões há dois comportamentos opostos que podem ser observados: a ação da força gravitacional atua favorecendo a troca térmica na posição da base, fazendo com que o tarugo seja pressionado sobre o molde, e o contato na interface metal/molde seja melhorado. Porém, no topo, a ação da força gravitacional, juntamente com a contração de

(a)
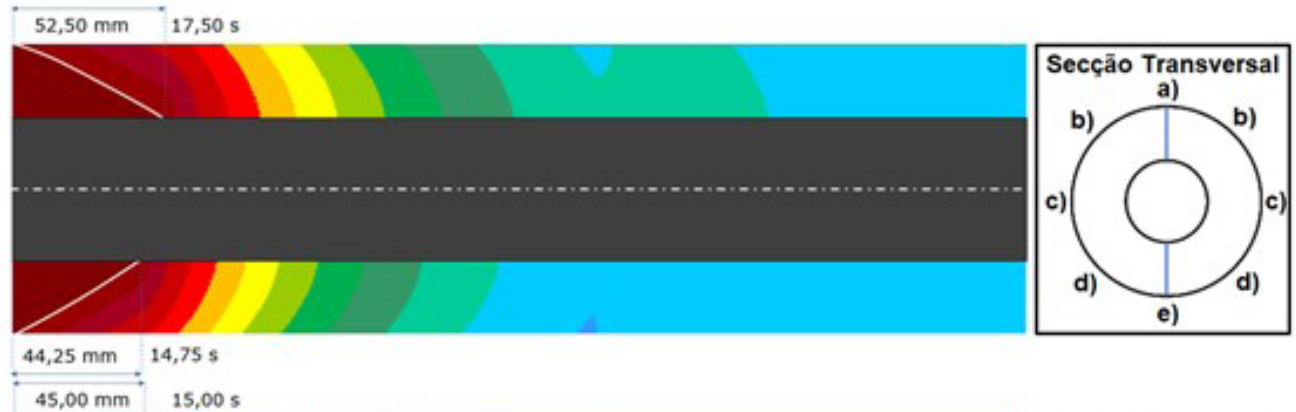

$45,00 \mathrm{~mm} \quad 15,00 \mathrm{~s}$

(b)
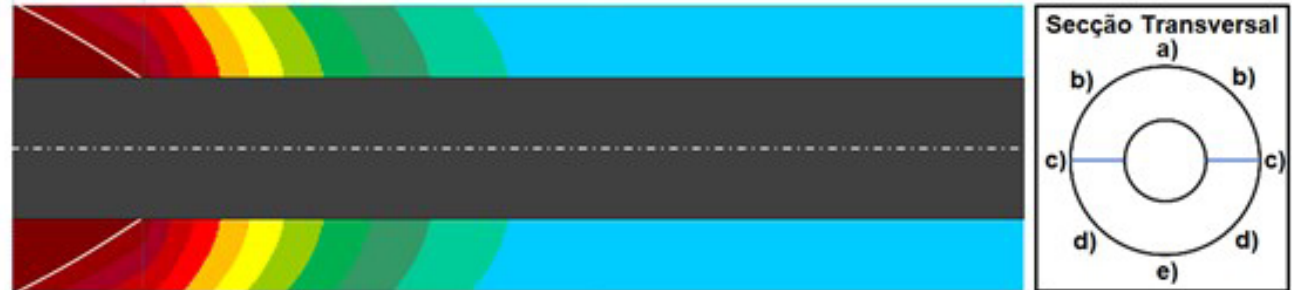

$45,00 \mathrm{~mm}, 15,00 \mathrm{~s}$

$48,00 \mathrm{~mm} 16,00 \mathrm{~s}$
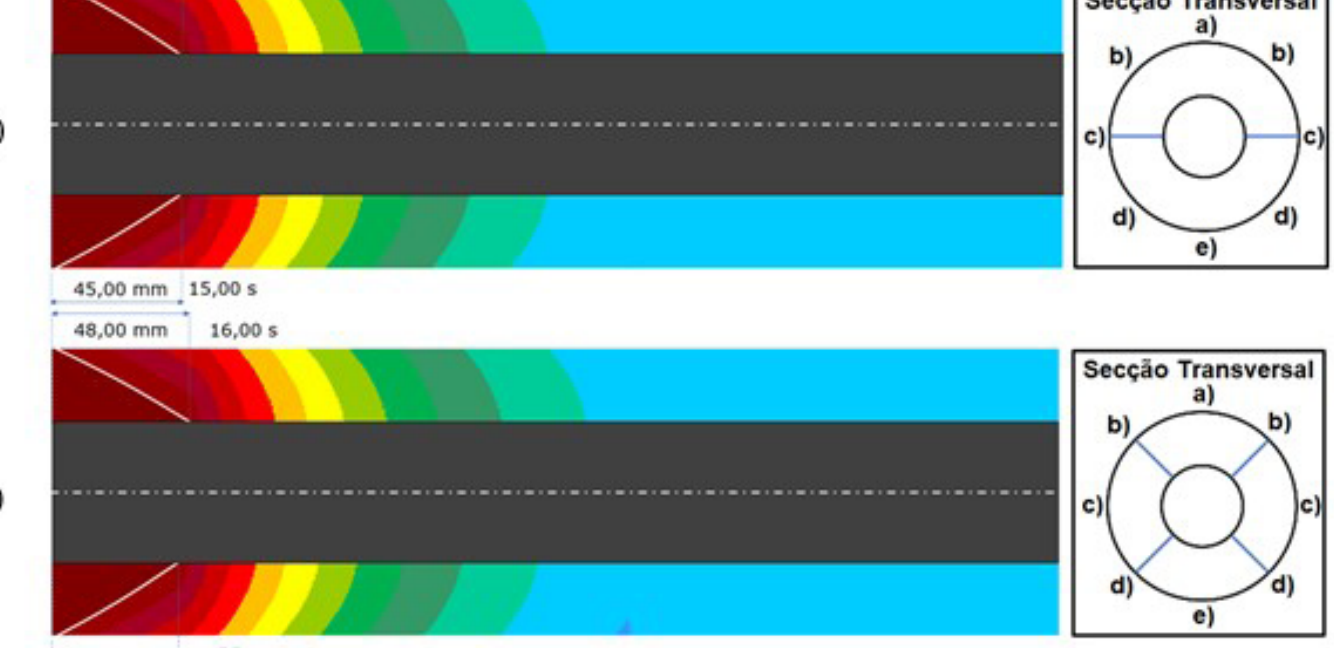

$44,25 \mathrm{~mm}, 14,75 \mathrm{~s}$

(c)

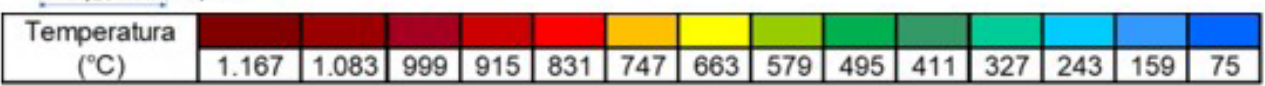

Figura 5. Perfil de temperaturas na secção longitudinal para a velocidade de $180 \mathrm{~mm} / \mathrm{min}$, referente às posições (a) topo e base, (b) laterais e (c) diagonal superior e inferior. 


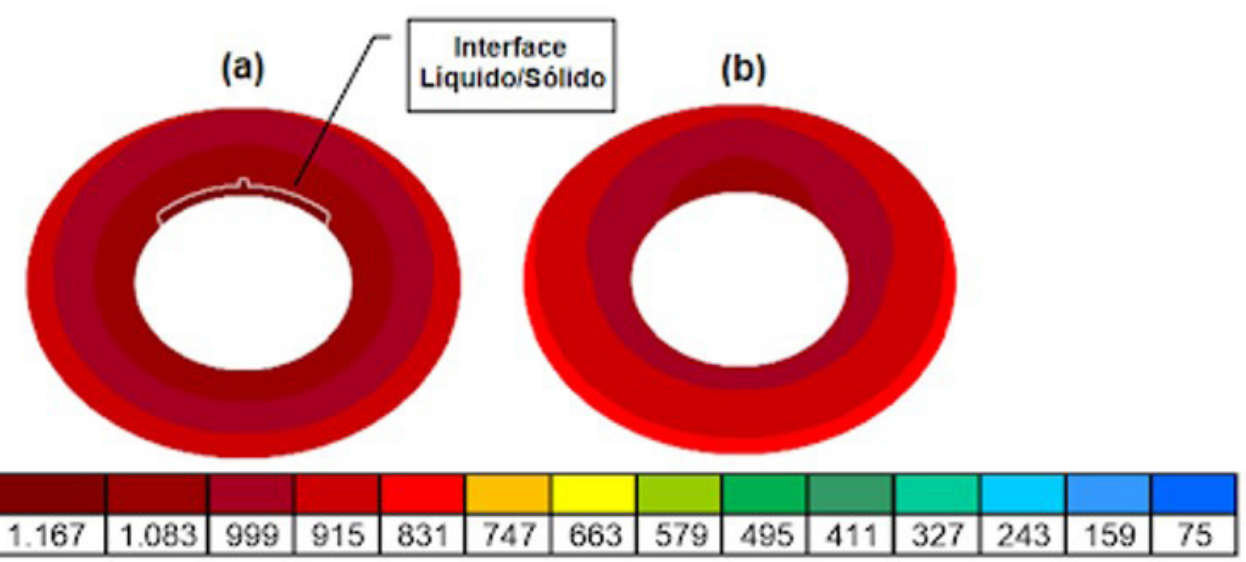

Figura 6. Isotermas na secção transversal em $44,25 \mathrm{~mm}$ (a) e $52,50 \mathrm{~mm}$ (b).

solidificação, faz com que o gap de ar seja mais acentuado, diminuindo a eficiência na troca térmica naquela região. Isto gera um gradiente térmico ao longo da superfície do lingote, impondo ao lingote diferentes tempos para que a interface líquido/sólido atinja o molde central. Como consequência, a partir do método inverso, os valores do coeficiente $h_{\mathrm{g}}$ foram determinados seguindo a tendência inversa de aumento à medida que se tomam posições em direção à base, pois para maiores de temperaturas, são esperados menores valores de $h_{g}$. Por fim, o mapeamento térmico no sentido longitudinal identificou a posição em que a interface líquido/sólido atinge o molde central, sendo visto que há uma diferença no tempo e posição para cada região do tarugo. Do ponto de vista da segurança operacional no ambiente industrial, levando-se em conta que a solidificação completa da secção transversal do tarugo ocorre a menos de $15 \%$ de deslocamento dentro do molde, pode ser dito que a velocidade de $180 \mathrm{~mm} / \mathrm{min}$ é segura para o processo, pois não haverá o risco de existir metal líquido a ser solidificado na saída do molde.

\section{Conclusão}

$\mathrm{O}$ equacionamento unidimensional proposto para o mapeamento térmico em uma secção longitudinal e extrapolação por progressão aritmética para a secção transversal, se mostrou eficiente para o entendimento do avanço da interface líquido/ sólido durante o lingotamento, a partir da determinação do coeficiente $h_{\mathrm{g}}$ para diferentes posições da superfície do lingote pelo método inverso IHCP. Com isso, foi possível confirmar que a secção transversal se solidificou completamente antes da saída do molde, servindo como parâmetro de controle para os engenheiros de produção responsáveis pela planta industrial onde o experimento foi realizado.

\section{Referências}

1 Straffelini G, Lutterotti L, Tonolli M, Lestani M. Modeling solidification microstructures of steel round billets obtained by continuous casting. ISIJ International. 2011;51:1448-1453.

2 Sha MH, Wang TM, Li L, Li TJ, Jin JZ. Numerical simulation of horizontal continuous casting process of round copper billet with electromagnetic stirring. International Journal of Cast Metals Research. 2011;24:197-202.

3 Aniceto PJ. Influência da velocidade de lingotamento no crescimento de grão colunares em tarugos ocos de cobre e correlações com a qualidade de tubos acabados [dissertação]. Santos: UNISANTA; 2017.

4 Rodriguez JM, Esteva A, Meza S. A note on the control of the solidification front in the continuous casting of copper tubes. Journal of Processing Technology. 1999;96:42-47.

5 Yan Z, Li X, Cao Z, Zhang X, Li T. Grain refinement of horizontal continuous casting of the CuNi10Fe1Mn alloy hollow billets by rotating magnetic field (RMF). Materials Letters. 2008;62:4389-4392.

6 Yan Z, Li X, Cao Z, Cai Q, Cao Z, Xialoi Z, et al. Study on horizontal electromagnetic continuous casting of CuNi10Fe1Mn alloy hollow billets. Materials \& Design. 2009;30:2072-2076.

7 Yu J, Jiang J, Ren Z, Ren W, Deng K. A new method of continuous casting of copper billets by a combination of AC current and magnetic fields. Materials \& Design. 2009;30:4565-4569.

8 Cheung N, Santos NS, Quaresma JMV, Dulikravich GS, Garcia A. Interfacial heat transfer coefficients and solidification of an aluminum alloy in a rotatory continuous casting. International Journal of Heat and Mass Transfer. 2009;52:451-459. 
9 Santos CA, Quaresma JMV, Garcia A. Determination of transient interfacial heat transfer coefficients in chill mold castings. Journal of Alloys and Compounds. 2001;319:174-186.

10 Incropera FP, Bergman TL, Dewitt DP. Fundamentos de transferência de calor e de massa. Rio de Janeiro: LTC; 2008.

11 Garcia A. Solidificação: fundamentos e aplicações. Campinas: UNICAMP, 2001.

12 Suresha KM, Prabhu KN. Effect of superheat, mold, and casting materials on the metal/mold interfacial heat transfer during solidification in graphite-lined permanent molds. Journal of Materials Engineering and Performance. 2004;13:619-626.

13 Bertelli F, Brito C, Meza ES, Cheung N, Garcia A. Inward and outward solidification of cylindrical castings: The role of the metal/mold heat transfer coefficient. Materials Chemistry and Physics. 2012;136:545-554.

14 Dusinberre GM. Numerical analysis of heat flow. New York: McGraw-Hill; 1949.

15 Beck JV. Nonlinear estimation applied to the nonlinear inverse heat conduction problem. International Journal of Heat and Mass Transfer. 1970;13:703-716.

16 Kreith F, Manglik RM, Bohn MS. Principles of heat transfer. 7th ed. Colorado: Cengage Learning; 2010.

17 Fluke Corporation. Manual Fluke Ti32. USA; 2020 [acesso em 6 nov. 2020]. Disponível em: https://assets. testequity.com/te1/Documents/pdf/manuals/Ti32-TiR32-M.pdf

Recebido em: 25 Jun. 2020

Aceito em: 5 Jan. 2021 